\title{
FORMACION DE UN PLANTEL BASE DE GANADO BOVINO CRIOLLO ARGENTINO PARA PRODUCCION LECHERA. EFECTO SOBRE LAS FRECUENCIAS GENICAS DE SEIS LOCI.
}

\author{
María Ripoli, Guillermo Giovambattis ta, Julio De Lúea, Fernando Dulout, \\ Centro de Inves tigaciones en Genética Básica y Aplicada (CIGEBA), \\ 60 y 118, CC 296, 1900 La Plata, Argentina, ggiovam@fcv.medvet.unlp.edu.ar. \\ Fernando Labarta, José Echenique, Marcelo Sánchez, Edgardo Carrizo, \\ Facultad de Ciencias Agrarias. Universidad Nacional de Jujuy. \\ Bolivia 1239.4600 San Salvador de Jujuy
}

Recepcion originales 17 de Mayo de 1999

\begin{abstract}
Formation of a foundation herd of Argentine Creole Cattle for milk production. Effect on six loci gene frequencies.

Key words: Creole cattle, Selection, Genetics Markers, Milk production.

With the purpose to establish a foundation group to carry out a dairy improvement plan for Argentine Creole cattle, thirty-eight dams and one sire belonging to this breed were selected. In order to evaluate such selection effect on six loci, polymorphisms of b-lactoglobulin, growth hormone, F13A, BoLA-DRB3, BoLA-DYA, and the microsatellite $D 23 S 5$ were characterized in the selected population (38 animals), as well as in unselected population (78 animals). The characterization of b-lactoglobulin, growth hormone, F13A, BoLA-DRB3, $B o L A-D Y A$ alleles was carried out through the techniques of PCR-RFLP, while the D23S5 variants were characterized by PCR-sequencing gel methodology. These results revealed a significant increase of $b-$ lactoglobulin B variant and non significant increase in D23S5 B variant. Whereas no significant difterences between the selected and unselected population were observed for gene frequencies of growth hormone F13A, BoLA-DRB3, BoLA-DYA.

The observed differences could be consequence of selection eftect. Family studies would be nessesary to confirm that the observed differencies were consequence of association between these molecular markers with quantitative trait loci (QTLs) for milk production.
\end{abstract}

\section{RESUMEN}

Palabras Claves: Ganado criollo, selcción marcadores genéticos,producción de leche.

Con el objetivo de establecer un plantel base de ganado Bovino Criollo Argentino para producción lechera, se seleccionaron treinta y ocho vientres y un toro pertenecientes a esta raza. Para poder evaluar el efecto de la selección sobre las frecuencias génicas, se caracterizaron los polimorfismos presentes en seis loci: blactoglobulina, hormona de crecimiento, F13A, BoLA-DRB3, BoLA-DYA, y en el microsatélite D23S5, tanto en la población seleccionada (38 animales), como en el rebaño no seleccionado (78 animales). La caracterización de b-lactoglobulina, hormona de crecimiento, F13A, BoLA-DRB3, BoLA-DYA se llevó a cabo a través de la técnica de PCR-RFLP, mientras que las variantes del microsatélite D23S5 se caracterizaron mediante PCR-electroforesis en geles de secuenciación. Los resultados evidenciaron un aumento significativo de la variante $\mathrm{B}$ de b-lactoglobulina, y un incremento no significativo de la variante $\mathrm{B}$ del microsatélite D23S5. Por otra parte las frecuencias génicas de los loci hormona de crecimiento, F13A, 
BoLA-DRB3, BoLA-DYA, no mostraron diferencias significativas entre la población seleccionada y la no seleccionada. Las diferencias observadas podrían ser consecuencia del efecto de la selección. Sin embargo, sería necesario realizar estudios familiares para confirmar que las diferencias observadas serían consecuencia de la asociación entre los marcadores moleculares analizados con loci de caracteres cuantitativos (QTLs) para producción lechera.

\section{INTRODUCCION}

Numerosos estudios realizados durante las últimas cuatro décadas, han puesto de manifiesto correlaciones estadísticamente significativas entre marcadores genéticos y caracteres de producción lechera. Dichos estudios han sido enfocados hacia el análisis de loci candidatos y/o hacia el rastreo del genoma mediante el uso simultáneo de un gran número de marcadores moleculares altamente polimórficos, tales como los microsatélites (Zwiauer, 1980; Beattie, 1994; Haley, 1995; Georges \& Andersson, 1996; Giovambattista et al. 1998). Se ha definido a los loci candidatos como aquellos genes que por su función biológica podrían participar en la expresión de un carácter cuantitativo. Pueden mencionarse a modo de ejemplo las proteínas de la leche, la prolactina $(P R L)$, la hormona de crecimiento $(G H)$, etc.

Existen evidencias bien documentadas que permiten correlacionar algunas de las variantes de las proteínas de la leche (k-caseína, b-caseína, $a_{\mathrm{S} 1}$-caseína, $\mathrm{a}_{\mathrm{S} 2}$-caseína, a-lactoglobulina y b-lactoglubulina) con el rendimiento y la composición del producto lácteo obtenido (e.g. McLean et al., 1984; Boland et al.., 1992; Lin et al, 1986; McLean y Schaar, 1989; Ng-Kwai-Hang et al, 1984, 1990; Cowan et al, 1990). También se han asociado las variantes alélicas de diversas hormonas ( $P R L$ y $G H)$ con caracteres de produción lechera (Cowan et al., 1990 ; Hoj et al., 1993; Yao et al., 1996a).

Además, cabe mencionar que varios genes del Complejo Principal de Histocompatibilidad pueden tener influencia, en forma directa o indirecta, sobre los niveles de producción (Batra et al., 1989; Beever et al. 1990; Stear et al, 1988; Weigel et al, 1990 ; Ostergärd et al, 1989; Mejdell et al, 1993, 1994a, 1994b). Por otra parte, en los últimos años, se han encontrado posibles asociaciones entre distintos microsatélites y caracteres de producción lechera (Georges et al, 1993; Ron et al, 1994; Georges et al, 1995; Spelman et al 1996 ; Ashwell et al, 1996; Vilkki et al 1997). Por lo tanto, el análisis del polimorfismo de los loci candidatos y de los microsatélites podría constituir una información útil para los programas de selección de ganado lechero.

La mayoría de los estudios basados en el análisis de marcadores genéticos se han llevado a cabo principalmente en razas seleccionadas para distintos fines productivos, donde la variabilidad se ve reducida en gran medida. Es decir, se están analizando las consecuencias de un largo proceso selectivo y no el proceso en sí mismo.

El ganado bovino Criollo Argentino (ACc), definido como el descendiente puro y directo de los vacunos introducidos por los conquistadores españoles en el territorio argentino (Primo, 1992), presenta un alto grado de variabilidad genética por no haber soportado una alta presión de selección artificial. Además, como consecuencia de muchos años de selección natural, el ganado ACc se ha adaptado a una amplia variedad de condiciones ambientales, desarrollando una gran variabilidad fenotípica, altos niveles de longevidad y fertilidad, además de una alta resistencia a enfermedades subtropicales (Sal Paz, 1986; $\underline{\text { Hansen, 1994). }}$

El objetivo del presente trabajo consistió en evaluar el efecto de la primera etapa de selección de bovinos Criollos Argentinos Lecheros sobre las frecuencias génicas de seis marcadores genéticos. Dos de ellos, $G H$ y b-lactoglubulina (b- $L G$ ), se han asociado con producción lechera, mientras que el BoLA-DRB3 ha sido 
correlacionado con la respuesta inmune y por lo tanto podría afectar, en forma indirecta, los niveles de producción. Los loci restantes, BoLA-DYA, F13A y D23S5 (MGTG7) no han sido asociados hasta el momento con cantidad y calidad composicional de la leche. De esta manera se amplió el estudio realizado en los mismos rebaños por Ripoli et al (1999), en el que se estimó el efecto de la selección sobre las frecuencias génicas de los loci k-caseína, $\mathrm{a}_{\mathrm{S} 1}$-caseína y prolactina

\section{MATERIALES Y METODOS}

\section{Poblaciones Estudiadas}

Rebaño General de animales no seleccionados. El rebaño general analizado correspondió a la población de la estación Zootécnica Subtropical ARROYO DEL MEDIO (EEAM), ubicada en el monte chaqueño de transición, en el sudeste de la provincia de Jujuy, Argentina (Sánchez Mera, 1989). Dicha estación recibió animales puros de la raza ACc provenientes de las localidades de El Pongo, Maimará, Lavayén, San Juan de Dios, Palma Sola en la provincia de Jujuy, y Anta en la provincia de Salta (Figura 1). Actualmente, la población de la EEAM cuenta con un plantel de aproximadamente 150 animales.

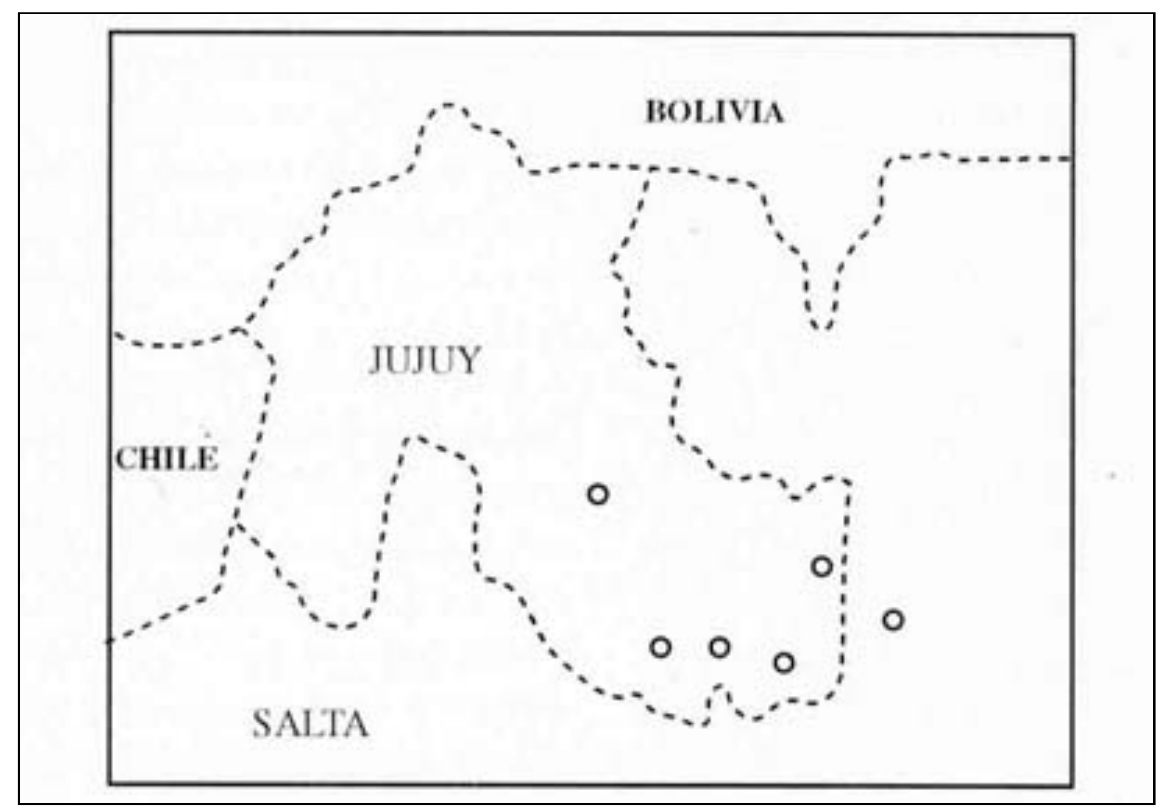

Figura 1: Ubicación geográfica de las localidades de Las Animas (1), Maimará (2), San Antonio (3), Estación Experimental El Remate (4), El Pongo (5), Lavallén (6), San Juan de Dios (7), Estación Zooté cnica Subtropical Arroyo del Medio (8), Anta (Salta) (9), y Palma Sola (10).

Selección y adquisición de animales para el proyecto lechero.Con el objetivo de establecer un plantel base para la selección de bovinos Criollos lecheros se seleccionaron 32 vientres y un toro de la EEAM. Por otra parte, fueron adquiridos 6

animales a productores locales de la provincia de Jujuy (4 de Las Animas, Dpto. de Tilcara y 2 de La Cabana, Dpto. de San Antonio). Actualmente, este plantel se encuentra localizado en la Estación Experimental El Remate (EEER).

Las hembras provenientes de las Estaciones Experimentales fueron seleccionadas según los siguientes criterios: pesos de los terneros al destete logrados por los vientres durante su vida productiva, biotipo, antecedentes genealógicos y otros antecedentes lecheros obtenidos en las Estaciones Experimentales. 
El sistema empleado para la compra de las vacas a productores consistió en la búsqueda de datos en distintos puestos de hacienda de los departamentos de San Antonio y Tilcara, con la finalidad de individualizar animales sujetos a ordeña y seleccionar algunas vacas y vaquillas para incorporarlas al plantel lechero. En este caso la selección se basó en los antecedentes suministrados por sus criadores, en el fenotipo, conformación y suspensión de las ubres; tamaño, orientación y pigmentación de los pezones; ausencia de pezones supernumerarios y temperamento de los animales (arrizo, 1995).

\section{Metodología Experimental}

Se obtuvieron 116 muestras de sangre periférica de bovinos de raza ACc pertenecientes a las poblaciones de EEAM (78 animales) y de EEER (38 animales). El aislamiento del ADN se llevó a cabo mediante la técnica de extracción con DNA ZOL, (Gibco BRL - life Technologies, Grand Island,N.Y,USA).

Técnicas de tipificación de los marcadores genéticos analizados: Hormona de crecimiento (GH): Para amplificar un fragmento de $404 \mathrm{pb}$ correspondiente al quinto exón del gen $G H$ se utilizó la técnica descrita por Yao et al. (1996a). Dicho fragmento posee el sitio de restricción polimórfico para la enzima Alu I que define los alelos A y B.

Alícuotas de los productos de amplificación (12 ml) fueron digeridos con la enzima de restricción Alu 1 (2,5 U) (Promega, Madison, WI). Los fragmentos de restricción fueron resueltos en geles de poliacrilamida 6\% (19:1) - TBE 1x, teñidos con bromuro de etidio y visualizados con luz UV.

b-lactoglobulina (b-LG): La caracterización del locus b-LG se realizó mediante la técnica de PCR-RFLP descrita por Medrano y Aguilar-Córdoba (1990). Esta metodología permitió amplificar un fragmento de 247 $\mathrm{pb}$ correspondiente al cuarto exón, en donde se encuentra el sitio de restricción polimórfico para la enzima Hae III que permite definir los alelos A y B.

Alícuotas de los productos de amplificación $(12 \mathrm{ml})$ fueron digeridos con la enzima de restricción Hae III, (2,5 U) (Promega, Madison, WI). Los fragmentos de restricción fueron resueltos en geles de poliacrilamida 6\% (19:1) - TBE Ix, teñidos con bromuro de etidio y visualizados con luz UV.

Subunidad A del factor de coagulación sanguínea (F13A): La caracterización del tercer exón del locus F13A se realizó mediante el método heminested-PCR-ACRS descrito por Park et al. (1995). Esta técnica permite identificar las variantes alélicas de este locus mediante la creación de un sitio de restricción artificial, utilizando un cebador que posee en el extremo $3^{\prime}$ una base no complementaria al ADN molde y que se ubica junto a la base polimórfica.

Alícuotas de los productos de amplificación (12 ml) fueron digeridos con la enzima de restricción Taq I (2,5 U) (Promega, Madison, WI). Los productos de amplificación fueron resueltos en geles de poliacrilamida $6 \%$ (19:1) - TBE 1x, teñidos con bromuro de etidio y observados con luz UV.

BoLA-DYA: La caracterización del segundo exón del gen BoLA-DYA se realizó mediante el método PCRACRS, el cual crea un sitio de restricción artificial para la enzima Hind III (Van der Poel et al 1990).

Alícuotas de los productos de amplificación (12 ml) fueron digeridos en forma simultánea con las enzima de restricción Hind III (2,5 U) y Hha I (2,5 U) (Promega, Madison, WI), para lo cual se utilizó el buffer universal MULTI-CORE (Promega, Madison, WI).

Los productos de amplificación fueron resueltos en geles de poliacrilamida $6 \%$ (19:1) - 1x TBE, teñidos con bromuro de etidio y visualizados con luz UV. 
BoLA-DRB3: La caracterización de los alelos del locus BoLA-DRB3 se realizó mediante la técnica heminested-PCR-RFLP descrita por Lewin et al (1992), la que permite amplificar un fragmento de $284 \mathrm{pb}$ correspondiente al segundo exón (sitio de reconocimiento del antígeno ARS) del gen.

Alícuotas de los productos de amplificación fueron digeridas, por separado, con las enzimas de restricción Rsa I, Bsty I y Hae III (2,5 U) (Promega, Madison, WI).

Los fragmentos de restricción fueron resueltos en geles de poliacrilamida al 6 u 8 \% (19:1) - TBE 1x, coloreados con bromuro de etidio y visualizados con luz UV.

Los alelos correspondientes a este locus fueron definidos mediante la combinación de los patrones obtenidos para las tres enzimas de restricción, según la nomenclatura propuesta por van Eijk et al..( 1992).

MGTG7 (D23S5): Para amplificar el fragmento correspondiente al microsatélite $M G T G 7$ (288 - $310 \mathrm{pb}$ ) se utilizaron los cebadores descritos por Bates et al., (1996).

Los alelos fueron resueltos en geles desnaturalizantes de poliacrilamida al 6\% (19:1) - TBE Ix, corridos a 50 watts durante 4 horas y revelados mediante la técnica de tinción con plata.

\section{Metodología Estadística}

Las frecuencias génicas y genotípicas se calcularon para cada uno de los loci analizados en el rebaño seleccionado para producción lechera (EEER) y en el rebaño general (EEAM).

El equilibrio de Hardy Weinberg se calculó a través del índice $\mathrm{F}_{1 \mathrm{~S}}$. El valor de significancia del índice $\mathrm{F}_{1 \mathrm{~S}} \mathrm{se}$ estimó mediante el Test de Probabilidad o el Test Exacto de Fisher descrito por Raymond y Rousset (1995).

La comparación de las frecuencias genotípicas observadas en los dos rebaños se llevó a cabo a través de tablas de contingencia, cuando las limitaciones del método lo permitieron.

La comparación entre las frecuencias génicas observadas en los dos rebaños fue realizada a través del análisis de Chi-Cuadrado, del método binomial de comparación de dos proporciones y del índice $\mathrm{F}_{\mathrm{ST}}$ modificado para comparar pares de poblaciones (Zar, 1984; Nei, 1987; Raymond y Rousset, 1995). Estos análisis se llevaron a cabo mediante la utilización de los programas Statgraphics, Plus, versión 3 (Manugistic, Inc., Rockville, MA, USA). Quatro (Borland International, USA) y Genepop (Raymond y Rousset, 1995) respectivamente. El valor significancia del índice $\mathrm{F}_{\mathrm{ST}}$ se estimó mediante el Test de Probabilidad o el Test Exacto de Fisher descrito por Raymond y Rousset, (1995).

Los niveles de diversidad genética se estimaron a través del número de alelos detectados $\left(\mathrm{n}_{\mathrm{a}}\right)$ y del índice de heterocigosidad esperada (he). Además se calculó la heterocigosidad promedio $\left(\mathrm{H}_{\mathrm{e}}\right)$ para los dos grupos, considerando tanto a los seis loci analizados en el presente trabajo, asi como a los genes $a_{\mathrm{S1}}$-caseína, kcaseína y PRL (Ripoli et al.. 1999). La comparación entre las $\mathrm{H}_{\mathrm{e}}$ estimadas para cada plantel se realizó mediante el método "t-Student" (ei, 1987). Las varianzas intra e interlocus de las $\mathrm{H}_{\mathrm{e}}$ se calcularon según las fórmulas propuestas por $\underline{\mathrm{Nei}(1987)}$.

\section{RESULTADOS}

En el cuadro 1 se detallan los valores de las frecuencias génicas y genotípicas estimadas para los loci de b$L G, G H$ y $F 13 A$. Dichos valores fueron calculados para el rebaño seleccionado de la EEER y el rebaño general de la EEAM. Las frecuencias génicas calculadas para el locus BoLA-DYA y para el microsatelite 
D23S5, se detallan en el cuadro II; mientras que las frecuencias génicas estimadas para el locus $B o L A-D R B 3$ se describen en el cuadro III.

La estimación del índice FIS evidenció que todos los loci analizados en ambas poblaciones se hallaban en equilibrio de Hardy-Weinberg, a excepción del microsatelite D23S5 que se encontró en desequilibrio en el rodeo EEAM (Cuadros 1, 2, 3). Cuando se calculó el índice FIS en foma conjunta para todos los loci, las dos poblaciones se encontraron en equilibrio $\left(\mathrm{c}^{2}\right.$ EEER $\left.=17,800 ; \mathrm{p}=0,599 ; \mathrm{c}^{2} \mathrm{EEEM}=17,0 ; \mathrm{p}=0,651\right)$.

La comparación de las frecuencias génicas y genotípicas correspondientes a los 2 grupos analizados puso de manifiesto que:

b- $L G$ : En el rebaño general las frecuencias génicas para las variantes A y B fueron similares, mientras que en el grupo seleccionado se observó un aumento del alelo $\mathrm{B}$ con respecto a la población original. Estas diferencias se encontraron en el límite de significación $\left(c^{2}=3,950 ; p=0,0469 ; Z=1,945 ; 0,05<p<0,10\right)$ (Cuadro 1), lo que fue confirmado mediante el índice $\mathrm{F}_{\mathrm{ST}}$ (Cuadro 4). La comparación de las frecuencias genotípicas correspondientes a ambos rodeos no evidenció diferencias significativas $\left(c^{2}=4,830 ; p=0,089\right)$.

Cuadro 1: Frecuencias génicas y genotípicas observadas para los loci de b-lactoglobulina, hormona de crecimiento y subunidad A del factor de coagulación sanguínea XIII en el rebaño general y el grupo seleccionado.

Table 1: Geneic and genotype frecuencies observed for loci of B-lactoglobuline groeth hormon and sun-unit a of blood clotting in the herd as well as the selected group.

$\beta$-lactoglobulina $(\beta-\mathrm{LG})$

Frecuencias Frecuencias

génicas genotípicas

$\mathrm{F}_{1 \mathrm{~S}}$

\begin{tabular}{lcccccc}
\hline Rebaño & alelo A & Alelo B & AA & AB & BB & \\
\hline Seleccionado & 0,250 & 0,750 & 0,071 & 0,357 & 0,572 & 0,085 \\
$\begin{array}{l}\text { (EEER) } \\
\text { General (EEAM) }\end{array}$ & 0,500 & 0,500 & 0,187 & 0,626 & 0,187 & $-0,220$ \\
\hline
\end{tabular}

Hormona de crecimiento $(\mathrm{GH})$

$\begin{array}{ccc}\begin{array}{c}\text { Frecuencias } \\ \text { génicas }\end{array} & \begin{array}{c}\text { Frecuencias } \\ \text { genotípicas }\end{array} & \mathrm{F}_{1 \mathrm{~S}}\end{array}$

\begin{tabular}{lcccccc}
\hline Rebaño & alelo A & alelo B & AA & AB & BB & \\
\hline $\begin{array}{l}\text { Seleccionado } \\
\text { (EEER) }\end{array}$ & 0,750 & 0,250 & 0,555 & 0,389 & 0,055 & 0,008 \\
General (EEAM) & 0,765 & 0,235 & 0,588 & 0,353 & 0,059 & $-0,050$ \\
\hline
\end{tabular}


Frecuencias génicas
Frecuencias

genotípicas
$\mathrm{F}_{1 \mathrm{~S}}$

\begin{tabular}{llllll}
\hline Rebaño & alelo $b$ & alelo B & bb & Bb & BB
\end{tabular}

Seleccionado

(EEEM)

$0,170 \quad 0,830 \quad 0,040$

0,250

0,710

0,121

General (EEAM)

0,110

0,890

0,030

0,170

0,890

0,126

$G H$ : En el rebaño general y en el seleccionado las frecuencias génicas para las variantes A y B fueron similares. Las diferencias entre los dos rodeos no fueron significativas $\left(\mathrm{c}^{2}-0,02 ; \mathrm{p}=0,878 ; \mathrm{Z}=0,131 ; \mathrm{p}>\right.$ 0,50 ) (Cuadro 1), lo que fue confirmado mediante el índice $\mathrm{F}_{\mathrm{ST}}$ (Cuadro 4). La comparación de las frecuencias genotípicas correspondientes a ambos rebaños no evidenció diferencias significativas $\left(\mathrm{c}^{2}=1,10\right.$; $\mathrm{p}=0,576)$.

F13A: Las frecuencias génicas para las variantes $\mathrm{B}$ y $\mathrm{b}$ fueron similares en los dos rebaños, no observándose diferencias significativas entre las frecuencias génicas $\left(\mathrm{c}^{2}-0,81 ; \mathrm{p}=0,368 ; \mathrm{Z}=0,679 ; \mathrm{p}=\right.$ 0,50) (Cuadro 1), 1o que fue confirmado mediante el índice $\mathrm{F}_{\mathrm{ST}}$ (Cuadro 4). La comparación de las frecuencias genotípicas correspondientes a ambos rebaños tampoco evidenció diferencias significativas $\left(\mathrm{c}^{2}=\right.$ $1,01 ; \mathrm{p}=0,603)$.

BoLA-DYA: Las cuatro variantes alélicas reportadas en bovinos se detectaron en ambos rebaños. El alelo G1G2 fue el más abundante en las dos poblaciones, con una frecuencia superior al $60 \%$, la variante G1A2 se detectó en ambos grupos con frecuencia del orden del 25\%, mientras que los alelos A1A2 y A1G2 fueron detectadas en las dos poblaciones con una frecuencia inferior al $5 \%$ (Cuadro 2). Las diferencias entre las frecuencias génicas estimadas para ambos rodeos resultaron ser no significativas $\left(c^{2}=1,45 ; 0,99>p>\right.$ 0,975), lo que fue confirmado mediante el índice $\mathrm{F}_{\mathrm{ST}}$ (Cuadro 4). La comparación de las frecuencias genotípicas correspondientes a ambas poblaciones tampoco evidenció diferencias significativas $\left(\mathrm{c}^{2}=1,370\right.$; $\mathrm{p}=0,712)$.

Cuadro 2: Frecuencias génicas observadas para el locus BoLA-DYA y para el microsatélite D23S5 en el rebaño general y el grup seleccionado.

Table 2: Gentic frequencis observed for locus Bola-Dya and for the microsatellite D2355 in the herdand selected group.

BoLA-DYA

Frecuencias génicas

$\mathrm{F}_{1 \mathrm{~S}}$

$\begin{array}{lccccc}\text { Rebaño } & \text { alelo } \mathrm{A}_{1} \mathrm{~A}_{2} & \text { alelo } \mathrm{G}_{1} \mathrm{~A}_{2} & {\text { Alelo } \mathrm{A}_{1} \mathrm{G}_{2}} & \text { alelo } \mathrm{G}_{1} \mathrm{G}_{2} & \\ \text { Seleccionado (EEER) } & 0,048 & 0,309 & 0,024 & 0,619 & 0,287 \\ \text { General (EEAM) } & 0,023 & 0,227 & 0,045 & 0,705 & 0,113\end{array}$


MGTG7 (D23S5)

Frecuencias génicas

$\mathrm{F}_{1 \mathrm{~S}}$

\begin{tabular}{lcccc}
\hline Rebaño & alelo A & alelo B & alelo C & \\
\hline Seleccionado (EEER) & 0,028 & 0,530 & 0,442 & 0,108 \\
General (EEAM) & 0,150 & 0,330 & 0,520 & $0-1,118^{*}$ \\
\hline
\end{tabular}

Cuadro 3: Frecuencias génicas observadas para el locus de BoLA-DRB3 en el rebaño general y el grupo seleccionado.

Table 3: Gentic frequencis observed for locus Bola-Dya and for the microsatellite D2355 in the herdand selected group.

BoLA-DRB3

Rebaño

\begin{tabular}{|c|c|c|}
\hline Frecuencia génica & $\begin{array}{c}\text { Seleccionado } \\
\text { (EEEM) }\end{array}$ & $\begin{array}{l}\text { General } \\
\text { (EEAM) }\end{array}$ \\
\hline$D R B 3.2 * 1$ & 0 & 0,006 \\
\hline$D R B 3.2 * 5$ & 0,023 & 0,110 \\
\hline$D R B 3.2 * 7$ & 0 & 0,006 \\
\hline$D R B 3.2 * 8$ & 0,023 & 0,041 \\
\hline$D R B 3.2 * 10$ & 0,159 & 0,145 \\
\hline$D R B 3.2 * 12$ & 0,045 & 0,023 \\
\hline$D R B 3.2 * 13$ & 0,023 & 0,012 \\
\hline$D R B 3.2 * 15$ & 0,250 & 0,215 \\
\hline$D R B 3.2 * 16$ & 0,068 & 0,035 \\
\hline$D R B 3.2 * 18$ & 0,159 & 0,076 \\
\hline DRB3.2*20 & 0,068 & 0,105 \\
\hline$D R B 3.2 * 21$ & 0,023 & 0,006 \\
\hline
\end{tabular}



DRB3.2*22
0,136
0,156
DRB3.2*23
0
0,006
DRB3.2*24
0,023
0,058
$\mathrm{F}_{1 \mathrm{~S}}$
0,036
0,033

D23S5: Las mismas vanantes alélicas se detectaron en ambos rebaños. Las frecuencias génicas estimadas para dichos alelos fueron distintas en las dos poblaciones, sin embargo las diferencias no fueron significativas $\left(c^{2}=5,23 ; p=0,073\right)$ (Cuadro 2), lo que fue confirmado mediante el índice $\mathrm{F}_{\mathrm{ST}}$ (Cuadro 4). La comparación de las frecuencias genotípicas correspondientes a ambas poblaciones tampoco evidenció diferencias significativas $\left(c^{2}=7,50 ; p=0,186\right)$.

BoLA-DRB3: Se detectaron un total de quince variantes alélicas en la EEAM y doce en la EEER. Los alelos $D R B 3.2 * 1, D R B 3.2 * 7$ y $D R B 3.2 * 23$ sólo se encontraron, con frecuencias muy bajas, en la población de la EEAM. El alelo $D R B 3.2 * 15$ fue mayoritario en ambos rebaños con una frecuencia del orden del $20 \%$. Los alelos $D R B 3.2 * 10, D R B 3.2 * 18, D R B 3.2 * 20$ y $D R B 3.2 * 22$ presentaron frecuencias considerables (entre 7\% y el 15\%) en las dos poblaciones. El resto de las variantes alélicas también fueron semejantes en ambos rebaños, con frecuencias menores al 10\%, a excepción del alelo $D R B 3.2 * 5$ cuya frecuencia fue del orden del $10 \%$ en la EEAM y del $2 \%$ en la EEER. Las diferencias entre las frecuencias génicas estimadas para ambos rebaños a través del índice FST resultaron ser no significativas (Cuadro 4).

\section{Cuadro 4: Valores estimados del índice $\mathrm{F}_{\mathrm{ST}}$, con su respectiva probabilidad y error} estándar, para los loci analizados.

Table 4: Index timate values with its probality and standard error, for analiged loci.

\begin{tabular}{lccc}
\hline \multicolumn{1}{c}{ Locus } & $\mathrm{F}_{\mathrm{ST}}$ & Probabilidad & Error estándar \\
\hline$\beta$-lactoglobulina & 0,096 & 0,065 & 0,002 \\
GH & 0,028 & 1,000 & 0,000 \\
F13A & 0,004 & 0,451 & 0,005 \\
BoLA-DYA & 0,009 & 0,432 & 0,008 \\
D23S5 & 0,020 & 0,075 & 0,005 \\
BoLA-DRB3 & 0,097 & 0,509 & 0,015 \\
\hline
\end{tabular}

Comparación de los niveles de diversidad genética entre EEER y EEAM: Los niveles de diversidad genética fueron estimados a través del cálculo de la $h_{\mathrm{e}} \mathrm{y}$ del $\mathrm{n}_{\mathrm{a}}$ para cada locus, así como también para el conjunto de ellos. Dichos valores, junto al error estandard de la $h_{e}$, se detallan en el cuadro 5 .

En ocho de los loci analizados se detectó el mismo número de alelos en las dos poblaciones, siendo la excepción el locus $B o L A-D R B 3$, ya que tres de sus alelos sólo se observaron en el rebaño general (Cuadro 5).

En cuanto a las $h_{e}$, seis de los loci presentaron valores levemente superiores en la EEER con respecto a la 
EEAM. En el resto de los genes la EEAM evidenció una mayor $\mathrm{h}_{\mathrm{e}}$. Al comparar los valores de $\mathrm{H}_{\mathrm{e}}$ estimados para las dos poblaciones, a través del método de "t-Student", no se observaron diferencias significativas $(\mathrm{t}=$ $0,011, \mathrm{p}>0,50)$.

\section{Cuadro 5: Estimación del $n_{a}, h_{\mathbf{e}} \mathbf{y}$ ES por loci en cada una de las poblaciones.}

Table 5: $n_{a}, h_{e} y$ ES estimation per loci on lach sampling.

\begin{tabular}{|c|c|c|c|c|c|c|}
\hline \multirow{3}{*}{ Locus } & \multicolumn{6}{|c|}{ Población } \\
\hline & \multicolumn{3}{|c|}{ EEAM } & \multicolumn{3}{|c|}{ EEER } \\
\hline & $\mathrm{n}_{\mathrm{a}}$ & $\mathrm{h}_{\mathrm{e}}$ & $\mathrm{ES}$ & $\mathrm{n}_{\mathrm{a}}$ & $\mathrm{h}_{\mathrm{e}}$ & ES \\
\hline$\alpha_{\mathrm{S} 1}$-caseina & 2 & 0,434 & 0,027 & 2 & 0,509 & 0,015 \\
\hline k-caseína & 2 & 0,503 & 0,005 & 2 & 0,476 & 0,039 \\
\hline$\beta-\operatorname{Lg}$ & 2 & 0,388 & 0,019 & 2 & 0,516 & 0,022 \\
\hline Prolactina & 2 & 0,035 & 0,019 & 2 & 0,078 & 0,070 \\
\hline $\mathrm{GH}$ & 2 & 0,370 & 0,084 & 2 & 0,387 & 0,078 \\
\hline F13A & 2 & 0,208 & 0,039 & 2 & 0,284 & 0,072 \\
\hline BoLA-DYA & 4 & 0,391 & 0,091 & 4 & 0,527 & 0,054 \\
\hline D23S5 & 3 & 0,611 & 0,023 & 3 & 0,559 & 0,035 \\
\hline BoLA-DRB3 & 15 & 0,987 & 0,004 & 12 & 0,934 & 0,022 \\
\hline Promedio (He) & 38 & 0,458 & & 36 & 0,460 & \\
\hline
\end{tabular}

\section{DISCUSION}

Baker y Manwell (1980), realizaron la primera clasificación de las razas bovinas basada en el análisis de marcadores genéticos. A este primer trabajo le sucedieron estudios realizados por otros autores, como por ejemplo, Medjugorac et al., (1994); MacHugh et al, (1994, 1997). El análisis de las relaciones entre las razas es posible si se asume que los marcadores genéticos utilizados son selectivamente neutros, y que las poblaciones están en equilibrio bajo deriva y migración, por lo que la similitud entre las razas refleja el ancestro común. Por lo tanto los procesos de selección no habrían modificado las frecuencias génicas originales, las que serían consecuencia de la historia particular de cada raza, de los movimientos migratorios y de la distribución geográfica actual (Baker y Manwell, 1980; Medjugorac et al., 1994). Es por esta razón que las distintas razas han podido ser agrupadas en base a sus frecuencias génicas, coincidiendo dichas agrupaciones con las realizadas mediante datos históricos y morfológicos.

Por otra parte, estudios cronológicos han evaluado el efecto de los procesos de selección sobre las frecuencias génicas. Para cumplir con dicho objetivo, se compararon las frecuencias alélicas de toros utilizados en inseminación artificial durante las décadas del 50 y del 60, con toros de la misma población utilizados durante la década del 80. Estos trabajos evidenciaron diferencias significativas entre los dos grupos analizados, a modo de ejemplo se pueden mencionar los genes de k-caseína y de ornitina descarboxilasa (Velmala et al, 1993; Yao et al., 1996b).

En 1999, Ripoli et al estudiaron las consecuencias de la formación de un plantel base de criollos lecheros sobre las frecuencias génicas de los loci k-caseína, $\mathrm{a}_{\mathrm{s} 1}$-caseína y prolactina. Dicho estudio evidenció diferencias significativas en las frecuencias génicas del locus de $\mathrm{a}_{\mathrm{s} 1}$-caseína. En el presente trabajo, se 
extendió el análisis a los loci $G H$, b- $L G, F 13 A$, BoLA-DRB3, BoLA-DYA y al microsatélite D23S5.

La comparación entre las frecuencias génicas del rodeo seleccionado de la EEER y del rodeo general de la EEAM, mostró diferencias en el límite de significación en el locus b- $L G$. Mientras que en el resto de los genes las diferencias observadas no fueron significativas. Este hecho indicaría que la formación del plantel base resultó en una modificación de las frecuencias génicas en los loci b- $L G$ y $D 23 S 5$. Sin embargo estas diferencias no fueron tan marcadas como las reportadas para el locus de $\mathrm{a}_{\mathrm{s} 1}$-caseína (Ripoli et al, 1999).

Las frecuencias génicas de una población pueden verse modificadas por factores tales como endogamia, deriva génica, selección, etc. Las diferencias observadas en el presente estudio, no serían consecuencia de la acción del primer factor mencionado. Esto puede justificarse mediante el análisis de los pedigrí, ya que ambos rebaños presentaron niveles equivalentes de endogamia.

Por otra parte, la selección sólo puede actuar si la sustitución de un alelo por otro implica una variación en la adaptación del animal (Bovenhuis y Weller, 1994). Un gran número de trabajos han estudiado el efecto del gen b- $L G$ sobre los caracteres de producción lechera en razas europeas altamente seleccionadas (Giovambattista et al, 1998). A nivel poblacional, estudios del comportamiento productivo, han puesto de manifiesto que la variante A está asociada con un mayor nivel de b-lactoglobulina y proteínas totales del suero de la leche, y con un menor contenido de caseínas y grasa (Boland et al, 1992). Por otra parte, los animales con genotipo b- $L G B B$ tienen un menor contenido de b-lactoglobulina y un mayor contenido de caseínas, proteínas totales y grasa en la leche, lo que resulta en mejores propiedades para la producción de queso (Tong et al, 1993). Además este genotipo ha sido correlacionado con un mayor rendimiento en litros de leche con respecto al genotipo AA, habiéndose observado para el genotipo AB un rendimiento intermedio (Jadot et al, 1992). En coincidencia con lo observado en las razas europeas, la selección de animales para producción lechera (EEER) produjo un aumento de la variante B que se encontró en el límite de significación.

La expresión diferencial de los alelos A y B de b- $L G$ ha sido observada en diferentes poblaciones de ganado bovino lechero (Aschaffenburg y Drewry, 1955; Cerbulis y Farrel, 1975; McLean et al., 1984; Kroeker et al, 1985; Ng-Kwai-Hang et al, 1987; Hill, 1993; Lum et al, 1997). Esto es debido a la presencia de sustituciones nucleotídicas, entre ambas variantes alélicas, en la región promotora de b- $L G$ que modifican la afinidad de los factores de transcripción (por ejemplo: AP-2) y por lo tanto los niveles de expresión. En los animales heterocigotos aproximadamente el $60 \%$ de la b-lactoglobulina corresponde a la variante A, mientras que el 40\% restante está contituída por la variante B (Graml et al., 1989; Wilkins et al, 1995; $\underline{\text { Lum et al. }}$, 1997).

En cuanto al gen $G H$, Lee et al (1996), estudiaron el sitio de restricción polimorfía) Alu I (exón 5) en un rebaño seleccionado y en otro control de la raza Holstein. A pesar que en el primer grupo, el genotipo Alu -/estaba asociado con una disminución en el valor de cría para la producción lechera, no observaron diferencias entre las frecuencias génicas de ambos grupos. Por otra parte, Hoj et al. (1993), agruparon bovinos pertenecientes a las razas Danesa y Noruega Rojas en dos grupos, según fuera alta o baja la producción de grasa en leche. Los animales fueron tipificados para el polimorfismo I/D (región 3') y para el sitio de restricción Msp I (intrón 3). Los resultados obtenidos indicarían que la selección sobre el carácter grasa en leche habría incrementado las frecuencias de los alelos D y Msp I (-). Finalmente, Yao et al. (1996a) analizaron el polimorfismo del gen GH en toros Holstein, a través de la técnica de SSCP (Single Strand Conformation Polymorphism). Dos de los fragmentos variables evidenciaron asociaciones significativas entre las variantes de $G H$ con mayor producción de leche, grasa y proteínas.

Sin embargo, en el presente trabajo las frecuencias génicas correspondientes al locus $G H(A l u \mathrm{I}+/-)$ no 
evidenciaron diferencias significativas entre el grupo seleccionado y el rebaño general. La ausencia de diferencias podría ser consecuencia de distintos factores. Si tenemos en cuenta que Yao et al.(1996a) reportaron la presencia de dos variantes de SSCP por cada uno de los alelos Msp I (+) y Alu I (+), este hecho podría ser una de las causas de los resultados obtenidos.

Por otra parte, dado que el ligamiento entre el QTL (Quantitative Trait Loci) y el marcador genético es incompleto, podrían existir en la población diferentes haplotipos marcador- $Q T L$. Así por ejemplo, el alelo $G H A$ podría estar ligado al alelo de alta producción del $Q T L$ en algunos animales, mientras que en otros estaría ligado a la variante de baja productividad del $Q T L$. Por lo tanto, los efectos estimados del $Q T L$ sobre el rasgo cuantitativo se verán sesgados por efecto de la recombinación. Por último, cabe mencionar que no necesariamente en todas las razas, se tienen que presentar las mismas asociaciones entre un marcador y un QTL.

Así por ejemplo, en la raza Danesa Roja la variante Msp I (-) fue asociada con mayor producción lechera, mientras que en la raza Holstein este alelo se asoció con baja producción de leche ( al., 1996a).

El Complejo Principal de Histo-compatibilidad (denominado Bovine Lymphocyte Antigen, BoLA), ocupa un rol central en la respuesta inmune. Este complejo es de gran importancia para la adaptación del individuo al medio, por lo que influye directa o indirectamente sobre los caracteres de producción. Numerosos estudios han demostrado la asociación entre el BOLA y caracteres de importancia fisiológica como fertilidad (Ostergärd et al., 1989; Mejdell et al., 1993, 1994 a,b), caracteres de producción, parámetros de crecimiento (Batra et al., 1989; Beever et al., 1990; Stear et al., 1988; Weigel et al., 1990), susceptibilidad o resistencia a enfermedades, como por ejemplo leucosis bovina, mastitis y parásitos intestinales (Lewin y Bernoco, 1986; Bernoco y Lewin, 1989; Lewin, 1989; Lundén et al., 1990; Schook et al., 1991; Zanotti et al., 1995; 1996). Estos estudios revelaron la importancia del análisis de estos marcadores, tanto para el estudio de la resistencia a enfermedades, como para los caracteres de producción (Lewin et al., 1991), ya que numerosas evidencias han demostrado que los animales portadores de las enfermedades antes mencionadas presentan una importante disminución en la producción (Yang et al., 1993). Entre los genes más estudiados se pueden mencionar el locus de Clase II BoLA-DRB3 y entre los loci de Clase I, el $B o L A$ A.

En el presente trabajo se analizaron tres loci pertenecientes al Complejo Mayor de Histocompatibilidad: $B o L A-D R B 3$, BoLA-DYA y D23S5, También fue incluido en el estudio el gen $F 13 A$ el que se encuentra, al igual que dicho complejo, en el BTA23. Los resultados obtenidos no evidenciaron diferencias significativas. Sin embargo, el valor estimado para el microsatelite D23S5 se encontró cercano al límite de significación.

La ausencia de diferencias para los loci BoLA-DYA y $F 13 A$ entre ambos rebaños, podría ser consecuencia de su ubicación en el cromosoma 23. Como se mencionó anteriormente los loci del $B o L A$ (Clase I y II) que han sido asociados con caracteres de importancia económica se encuentran en la región 2 del BTA23, mientras que el $B o L A-D Y A$ se encuentra cercano al centrómero, y el $F 13 A$ próximo al telómero. Por lo tanto la ausencia de diferencias podría deberse a que hasta el momento, en ninguna de estas dos regiones han sido reportados QTLs, y por otra parte a la gran distancia (cM) existente entre el BoLA-DYA y el $F 13 A$ con respecto a los loci de Clase I y II.

Con respecto al locus $B o L A-D R B 3$ y al microsatelite $D 23 S 5$, la formación del plantel base de criollos lecheros sólo modificó las frecuencias génicas de éste último, a pesar de encontrarse ambos estrechamente ligados. Este hecho podría deberse al gran número de alelos detectados para el BoLA-DRB3 con respecto al D23S5. 
A su vez, el número reducido de animales que conforman el plantel seleccionado hace que existan muchas clases representadas por pocos individuos, lo que dificulta la comparación de los dos grupos analizados.

Con respecto a la heterocigocidad, existen evidencias que en razas europeas altamente seleccionadas hay pérdida de variabilidad (e.g. MacHugh et al., 1997). En la población de la EEER no se produjo disminución de la heterocigocidad con respecto al rebaño general, al menos en esta primera etapa de selección. Sería de interés analizar las siguientes generaciones seleccionadas para producción lechera, para evaluar si los niveles de diversidad genética se mantienen a lo largo del proceso de selección.

Finalmente, la existencia de correlación entre las variantes alélicas del locus b- $L G$, así como para el locus $\mathrm{a}_{\mathrm{S} 1}$-caseína reportado anteriormente (Ripoli et al., 1999), con una mayor producción lechera en la raza ACc, debería confirmarse aumentando el número de animales caracterizados y a través de estudios familiares, en los que se relacionen dichos marcadores genéticos con los caracteres cuantitativos. De esta manera, podría determinarse la presencia de haplotipos característicos para dicha raza y confirmar que las diferencias observadas en el presente trabajo se deberían al proceso de selección y no a un error de muestreo.

\section{BIBLIOGRAFIA}

ASCHAFFENBURG R. y J. DREWRY. 1955. Ocurrence of different betalactoglobulins in cow's milk. Nature (Lond.) $176: 218$.

ASHWELL, M.S., C.E. REXROAD JR, R.H.MILLER y P.M. VANRADEN. 1996. Mapping economic trait loci for somatic cell score in Holtein cattle using micosatellites markers and selective genotyping. Animal Genetics $27: 235-242$.

BAKER, A.C.M. and MANWELL, C. 1980. Chemical classification of cattle. I. Breed groups. Anim. Blood Grps Biochem. Genet. 11:127-150.

BATES, S., C. PETERSON-KNABE, T. HOLM, H. VAN HAERINGEN, K. LANCE, J. ZIEGLE, D. HEYEN, Y. DA Y H. LEWIN. 1996. Exclusion probabilities of 22 bovine microsatellites markers in fluorescent multiplexes for automated parentage verification. Proceeding of the XXV International Society for Animal Genetics 28.

BATRA, T. R., A. J. LEE, R. S. GAVORA y M. J. STEAR. 1989. Class I alleles of the bovine major histocompatibility system and their association with economic traits. J. Dairy Sci. 72:2115-2124.

BEATTIE, C. 1994. Livestoek genome maps. TIG 10 : 334 - 338.

BEEVER, J.E., P.D. GEORGE, R.L. FERNANDO, C.T. STORMONT y H.A. LEWIN. 1990. Association between genetic markers and growth and carcass traits in a paternal half-sib family of Angus cattle. J. Anim. Sci. $68: 337$ - 344.

BERNOCO, D. y H.A. LEWIN. 1989. The bovine lymphocyte antigen (BoLA) system: Importance andrelatioship to disease in cattle. Rev. Bras. Genet. 12(3) : 107 - 122.

BOLAND, M.L, J.P. HILL y L.K. CREAMER. 1992. Genetic manipulation of milk proteins \& its consequences for the dairy industry. Australian Biotechnology 2(6) : 355 - 360 . 
BOVENHUIS, H. and WELLER, J.I. 1994. Mapping and analysis of dairy cattle quantitative trait loci by maximum likelihood methodology using milk protein genes as genetic markers. Genetics 137:267-280.

CARRIZO, E.N. 1995. Informe de avance del proyecto SeCTER/UNju A-49.1. Mejoramiento del Bovino Criollo como Productor de Leche en la Región Subtropical del NOA.

CERBULIS, J. y H. M. FARREL, JR. 1975. Compositions of milks of dairy cattle. I. Protein, lactose, and fat contents and distribution of protein fraction. J. Dairy Sci. $58: 817$.

COWAN, C. M., M. R. DENTINE, R. L. AX y L. A. SCCHULER. 1990. Structural variation around prolactin gene linked to quantitative traits in an elite Holstein sire family. Theor. Appl. Genet. 79: 577 - 582.

GEORGES M., D. NIELSEN, M. MACKINNON, A. MISHRA, R. OKINOTO, A.T. PASQUINO, L.S. SARGEANT, A. SORENSEN, M.R. STEELE y X. ZHAO. 1995. Mapping quantitative trait loci controlling milk production in dairy cattle by exploting progeny testing. Genetics $139: 907-920$.

GEORGES M., M. LARTHROP, A. B. DIETZ, A. LEFORT, F. LIBERT, A. MISHRA, D. NIELSEN, L. S. SARGEANT, M. R. STEELE, X. ZHAO, H. LEIPOLD y J. E: WOMACK. 1993. Microsatellite mapping of the gene causing weaver disease in cattle will allow the study of an associated QTL. Proc. Nati. Acad. Sci. 90: 1058-1062.

GEORGES, M.y L. ANDERSSON (1996) Livestock genomics comes of age. Genome Research 907-921.

GIOVAMBATTISTA G., M.V. RIPOLI, P. PERAL GARCÍA, J.C. DE LUGA, EN. DULOUT. 1998. Estado actual del mapeo de QTLS para producción lechera. Agrosur. 26: 78-96.

GRAML, R., G. WEISS, J. BUCHBERGER y F. PIRCHNER. 1989.

Different rates of synthesis of whey protein and casein by alleles of the beta-lactoglobulin and alpha-s1-casein locus in

cattle. Genet. Sel. Evol. $21: 547$.

HALE Y, C. S. 1995. Livestoek QTLs-bringing home the bacon?. TIG 11(12) : 488 - 492.

HANSEN,E.W. 1994. Ganadería bovina de raza criolla en el noroeste argentino. Universidad Nacional de Jujuy, San Salvador de Jujuy, Argentina. 150 pp.

HILL, J.P. 1993. The relationship between (-lactoglobulin phenotypes and milk composition in New Zealand dairy cattle. J. Dairy Sel. $76: 281$.

HOJ, S., M. FREDHOLM, N. LARSEN y V.H. NIELSEN. 1993. Growth hormone gene polymorphism associated with selection for milk fat production in lines of cattle. Animal Genetics 24 :91-96.

JADOT, M., J. LALOUX, A. BURNY y R. KETTMANN. 1992. Detection of bovine -lactoglobulin genomic variants by the polymerase chain reaction method and molecular hybridization. Animal Genetics 23(1) : $77-80$.

KROEKER, E. M., K. F. NG-KWAI-HANG, J. F. HAYES y J. E. MOXLEY. 1985. Effect of (lactoglobulin variant and enviromental factors on variation in the detailed composition of bovine milk serum proteins. J. Dairy Sci. $68: 1637$. 
LEE, B.K., G.F. LIN, B.A. CROOKER, M.P. MURTAUGH, L.B. HANSEN y H. CHESTER-JONES. 1996. Association of somatotropin (BST) gene polymorphism at the 5th exon with selection for milk yield in Holstein cows. Domestic Animal Endocrinology 13(4) : 373-381.

LEWIN, H. A. y D. BERNOCO. 1986. Evidence for BoLA linked resistance and susceptibility to subclinical progression of bovine leukaemia virus infection. Animal Genetics 17 : 197 - 207.

LEWIN, H.A. 1989. Disease resintance and immune response genes in cattle: strategies for their detection and evidence of their existence. J. Dairy Sci. 72: 1334 - 1348.

LEWIN H. A., P. A. CLAMP, J. E. BEEVER y L. B. SCHOOK.1991. Mapping genes for resistance to infectious diseases. In "Gene mapping : Techniques and applications" (L. B. Schook, H. A. Lewin, and D. G. McLaren, Eds.), pp. 283-303, Dekker, New York.

LEWIN, H.A., K. SCHMITT, R. HUBERT, M.J.T. VANELTKy N.ARNHEIM. 1992. Close linkage between bovine prolactin and BoLA-DRB3 genes: Genetics mapping in cattle by single sperm typing. Genomics $13: 44-48$.

LIN, C.Y.; MC. ALLISTER, A.J.; NG-KWAI-HANG, K.F. and HAYES, J.F. 1986. Effects of mik protein loci on first lactation production in dairy cattle. Journal of Dairy Sciences 69:704-712.

LUM L. S., DOVC P. y J. F. Medrano. 1997. Polymorphisms of bovine (-lactoglobulin promoter and differences in the binding aftinity of activator protein-2 transcripton factor. J. Dairy Sci. 80: 1389-1397.

LUNDÉN , A., S. SIGURDARDÓTTIR, I. EDFORS-LILJA, B. DANELL, J. RENDEL y L. ANDERSSON. 1990. The relationship between bovine major histocompatibility complex class II polymorphism and disease studied by use of bull breeding values. Animal Genetics $21: 221-232$.

MACHUGH, D. E., R. T. LOFTUS, D. G. BRADLEY, P. M. SHARP y P. CUNNINGHAM. 1994. Microsatellite DNA variation within and among European cattle breeds. Proc, R. Lond. B 256 :25-31.

MACHUGH, D. E., M. D. SHRIVER, R. T. LOFTUS, P. CUNNINGHAM Y D. G. BRADLEY. 1997. Microsatellite DNA variation and the Evolution, Domestication and Phylogeography of Taurine and Zebu Cattle. Genetics $146: 1071$ - 1086.

MCLEAN, DM, E.R.B. GRAHAM, R.W. PONZONI y H.A. MCKENZIE. 1984. Effects of milk protein genetic variants on milk yield and composition. J. Dairy Sci. 73 :2647-2656.

MCLEAN, D.M. y J. SCHAAR. 1989. Effects of -lactoglobulin and -casein genetic variants and concentration on syneresis of gels from renneted heated milk. J. Dairy Research $56: 296$.

MEDJUGORAC, L; KUSTERMANN, W.; LAZAR, R; RUSS, I. and PIRCHNER, F. 1994. Markerderived phylogeny of European cattle supports demic expansion of agriculture. Animal Genetics 25:19-27.

MEDRANO, J.F. y E. AGUILAR-CORDOBA. 1990. Polymerase chain reaction amplification of bovine blactoglobulin genomic sequences and identification of genetic variants by RFLP analysis. Biotechnology 1(1): 73-77. 
MEJDELL, C.M., $\varnothing$. LIE, H. SOLBU, E.F. ARNET y R.L. SPOONER. 1993. Associations of bovine lymphocyte antigens with milk and meat production traits of Norwegian cattle. J. Dairy Sci. 76:3778 - 3784.

MEJDELL, C.M., H. SOLBU, R.L. SPOONER, E.F. ARNET y $\varnothing$. LIE. 1994a. Changes in BoLA Class I aliele frequencies over years in norwegian Breeding bulls, and the association of BoLA with perfomance traits in young bulls. Acta Agric. Scand., Sect. A, Animal Sci. 44 : 73-78.

MEJDELL, CM, O. LIE, H. SOLBU, E.F. ARNET y R.L. SPOONER. 1994b. Association of major histocompatibility complex antigens (BoLA-A) with AI bull progeny test results for mastitis, ketosis and fertility in Norwegian cattle. Animal Genetics 25(2) : 99 - 104.

NEI, M. 1987. Molecular evolutionary genetics. Columbia University press. New York. pgs. 512.

NG-KWAI-HANG, K. F. , J. F. RAYES, J.E. MOXLEY y H. G. MONARDES.

1987. Variation in milk protein concentrations associated with genetic polymorphism and enviromental factors. J. Dairy Sci. $70: 563$.

NG-KWAI-HANG, K. F, G. MONARDES y J.FRAYES. 1990.Associationbetween genetic polymorphism of milk proteins and traits during three lactations. J. Dairy Sci. $73: 3414-3420$.

NG-KWAI-HANG, K.F; RAYES, J.F.; MOXLEY, J.E. and MONARDES, G. 1984. Association of genetic variants of casein and milk serum proteins with milk, fat and ptotein production by dairy cattle. $\mathrm{J}$. Dairy Sel. 67(4):835-840.

ØSTERGÁRD, H., B. KRISTENSEN y S. ANDERSEN. 1989. Investigacions in farm animals of associationss between the MHC system and disease resistance and fertility. Livest. Prod. Sci. $22: 49$ - 67.

PARK CH., I. RUSS, Y. DA \& H. A. LEWIN. 1995. Genetic mapping of F13 A to BTA23 by sperm typing: Difference in recombination rate between bulls in the DYA-PRL interval. Genomics 27:113-118.

PRIMO, A.T. 1992. El ganado bovino ibérico en las Américas: Quinientos años después. Arch.Zootec. (extra):421-432.

RAYMOND M, ROUSSET F .1995. GENEPOP 1.2: a population genetics sotfware for exact test and ecumenicism. J. Hered. 86, 248-249.

RIPOLI, M.V., G. GIOVAMBATTISTA, T.C. DE LUGA, F. LABARTA, J. ECHENIQUE, S. CASAS, E. CARRIZO, M. SÁNCHEZ MERA y EN. DULOUT. 1999. Formación de un plantel base de ganado bovino Criollo Argentino para producción lechera. Efecto sobre las frecuencias génicas de los loci de kcaseína, $\mathrm{a}_{\mathrm{s} 1}$-caseína y prolactina. Arch. Zootec. 48: (en prensa).

SAL PAZ, F.P. 1986. El ganado Criollo Argentino definiciones y características. En Primera Jornada Nacional de Ganado Bovino Criollo 1:3-7.

SANCHEZ MERA, M.G. 1989. Avances en un proyecto Agrosilvopastoril. Memorias X Reunión del Grupo Técnico Regional del Cono Sur en Mejoramiento y Utilización de los Recursos Forrajeros del Área Tropical y Subtropical. Talleres Gráficos INTA. Chile 460 B.A.. Pag. 21. 
SCHOOK, L.B., H.A. LEWIN y D.G. MCLAREN. 1991. Gene mapping techniques and applications. M. Dekker, inc. N. Y. USA. several production characteristics in three Danish cattle breeds. Acta Agrie Scandll: 163-196.

SPELMAN, R.T., W. COPPIETERS, J.A.M. VAN ARENDONK y H. BOVENHUIS. 1996. Cuantitativo traits loci analysis for five milk production traits on chromosomes six in the duch Holstein-Friesian population. Genetics (in press).

STEAR, M.J., T.S. POKORNY, N.E. MUGGLI y R.T. STONE. 1988. The relationships of birth weight, preweaning gain and postweaning gain with the bovine major histocompatibility system. J. Anim. Sci. 67 : 641 - 649 .

TONG, P S., S. VINK, N. Y. FARKYE y J. F. MEDRANO. 1993. Effects of genetic variants of mik protein on the yield of Cheddar cheese. Page 179 in Cheese Yield and factors Affecting Its Control. Int. Dairy. Fed., Cork, Ireland.

VAN DER POEL, J.J., M.A. GROENEN, R.LM. DLTKHOF, D. RUYTER Y MJ. GIPHART 1990. Denucleotide sequence of the bovine MHC class II alpha gene: DRA, DQA, and DYA. Inmunogenetics 31:29-36.

VAN EIJK M. J. T., J. A. STEWART-HAYNES \& H. A. LEWIN. 1992. Extensive polymorphism of BoLA -DRB3 gene distinguished by PCR-RFLP Animal Genetics 23: 483-496.

VELMALA, R.; MÄNTYSAARI, E.A. and MAKI-TANILA, A.. 1993. Molecular genetic polymorphism at the -casein and -lactoglobulin loci in Finnish dairy bulls. Agrie, Sel. Finí. 2:431-435.

VILKKI, H. J., D. J. de KONING, K. ELO, R. VELMALA, A. MAKI-TANILA. 1997. Multiple marker mapping of quantitative trait loci of Finnish dairy cattle by regression. J. Dairy Sel. 80(1): 198-204.

WEIGEL, K.A., A.E. FREEMAN, M.E. KEHRLI JR, M.J. STEAR y D.H. KELLEY (1990) Association of class I bovine lynphocyte antigen complex alleles with health and production traits in dairy cattle. L Dairy Sel. $73: 2538-2546$.

WILKINS, R. L, H. W. DAVEY, T. T. WHEELEER y C. A. FORD. 1995. Differential expression of blactoglobulin alleles A and B in dairy cattle. Page 189 in Intracellular Signaling in the Mammary Gland. C. L Wilde, M. Peaker, C. H. Knight, de. Plenum Press, New York, NY.

YANG, DA, D.S. ROGER, LA. STEWART y H. LERWIN. 1993. Milk and fat yields decline in bovine leukemia virus-infected Holstein cattle with persisten! lymphocytosis. Proc. Natl. Acad. Sel. USA. 90 : 6538-6541.

YAO J, S.E AGGREY, D. ZADWORNY, J.F. HAYES y U. KUHNLEIN. 1996a. Sequence variations in the bovine growth hormone gene characterized by single-strand conformation polymorphism (SSCP) analysis and their association with millk production traits in Holsteins. Genetics 144: 1809- 1816.

YAO, J.; ZADWORNY, D; KUHNLEIN, U.; AGGREY, S.E. and HAYES, J.F. 1996b. A Msp I polymorphism in the bovine ornithine decarboxylase gene and its possible association with selection for milk production in Holstein bulls. Animal Genetics 27:283-284. 
ZANOTTI, M., M. LONGERI M., M. POLLI, G. CERIOTTI y G. POLI. 1995. BoLA class II polymorphism andimmune response to Mycobacterium bovis antigens in vitro. J. Anim. Breed. Genet. 112: 391-400.

ZANOTTI, M., G. POLI, W. PONTI, M. POLLI, M. ROCCHI, E. BOLZANI, M. LONGERI, S. RUSO, H.A. LEWIN y M.J.T. VAN EIJK. 1996. Association of BoLA class II hplotypes with subclinical progression of bovine leukaemia virus infection in Holstein-Friesian cattle. Animal Genetics 27 :337-341.

ZAR J, H. 1984. Biostatistical Analysis. Pentice-Hall inc. Englewood Cliffs, N.J., USA. pp. 718.

ZWIAUER, D. 1980. Beziehungen zwischen biochemischen Markergenen und leistungseigenschaften bein Rind. Fortschr Tierz Züchtungsbiol 2. Parey, Hamburg Berlin. 